\title{
Issuer Credit Quality and the Price of Asset-Backed Securities
}

\author{
Oliver Faltin-Traeger \\ Columbia Business School \\ 3022 Broadway, Uris Hall \#311 \\ New York, NY 10027 \\ (212) 854-2836 (phone) \\ (212) 932-2359 (fax) \\ ohf2101@columbia.edu \\ Kathleen W. Johnson \\ Federal Reserve Board \\ Washington, DC 20551 \\ (202) 452-3644 (phone) \\ (202) 728-5887 (fax) \\ Kathleen.w.johnson@frb.gov \\ Christopher Mayer \\ Columbia Business School \& NBER \\ Visiting Scholar, Federal Reserve Bank of New York \\ 3022 Broadway, Uris Hall \#101 \\ New York, NY 10027 \\ 212-854-4221 (phone) \\ 212-932-0545 (fax) \\ cm310@columbia.edu
}

Mortgage Market and the Financial Crisis

Presiding: Nancy Wallace

Discussant: Nancy Wallace 
Preliminary and Incomplete - Please do not quote or cite.

\section{Appendix 1: ABS Sample Characteristics}

\begin{tabular}{lcc} 
& Securities & Dollar \\
Volume \\
\hline Collateral Type & $100 \%$ & $100 \%$ \\
Auto loans & $1.9 \%$ & $8.6 \%$ \\
CDOs & $3.5 \%$ & $5.2 \%$ \\
CMBS & $3.1 \%$ & $6.8 \%$ \\
Credit Cards & $1.5 \%$ & $10.5 \%$ \\
Equipment & $0.4 \%$ & $0.8 \%$ \\
Home equity & $55.6 \%$ & $28.7 \%$ \\
Man. Housing & $0.4 \%$ & $0.5 \%$ \\
RMBS & $32.1 \%$ & $32.5 \%$ \\
Student Loans & $1.4 \%$ & $6.3 \%$ \\
\hline Coupon Type & $100 \%$ & $100 \%$ \\
Floating rate & $63.1 \%$ & $69.6 \%$ \\
Fixed rate & $36.9 \%$ & $30.4 \%$ \\
\hline Trust Type & $100 \%$ & $100 \%$ \\
Mortgage Loan Trust & $50.2 \%$ & $51.2 \%$ \\
Other Trust & $49.8 \%$ & $48.8 \%$ \\
\hline Initial rating & $100 \%$ & $100 \%$ \\
AAA & $57.6 \%$ & $92.0 \%$ \\
AA & $11.9 \%$ & $2.9 \%$ \\
A & $11.6 \%$ & $2.6 \%$ \\
BBB & $11.8 \%$ & $1.9 \%$ \\
BB and below & $7.1 \%$ & $0.6 \%$ \\
\hline Parent Rating & $100 \%$ & $100 \%$ \\
AAA & $1.0 \%$ & $1.4 \%$ \\
AA & $22.3 \%$ & $22.2 \%$ \\
A & $44.0 \%$ & $43.9 \%$ \\
BBB & $9.0 \%$ & $8.4 \%$ \\
BB and below & $8.7 \%$ & $8.3 \%$ \\
NR & $15.1 \%$ & $15.8 \%$ \\
\hline Deal Participants & $100 \%$ & $100 \%$ \\
Sponsor $=$ servicer & $26 \%$ & $27 \%$ \\
Sponsor $=$ servicer $=$ underwriter & $18 \%$ & $15 \%$ \\
Servicer or underwriter unidentified & $25 \%$ & $25 \%$ \\
Sponsor $\neq$ servicer & $31 \%$ & $33 \%$ \\
\hline Sponsor Diversification & $100 \%$ & $100 \%$ \\
Collateral types issued $>4$ & $26 \%$ & $71 \%$ \\
Collateral types issued $\leq 4$ & $74 \%$ & $29 \%$ \\
\hline \hline Total & 99.019 secs. & $\$ 5,211 \mathrm{bn}$. \\
\hline & & \\
\hline
\end{tabular}


Preliminary and Incomplete - Please do not quote or cite.

\section{Appendix 2: Mean Coupon Spreads}

\begin{tabular}{lc}
\hline & $\begin{array}{c}\text { Average spread } \\
\text { (ppt.) }\end{array}$ \\
\hline Collateral Type & \\
Auto loans & 0.14 \\
CDOs & 1.54 \\
CMBS & 0.33 \\
Credit Cards & 0.94 \\
Equipment & 0.10 \\
Home equity & 0.81 \\
Man. Housing & 2.00 \\
RMBS & 0.86 \\
Student Loans & 0.44 \\
\hline Coupon Type & \\
Floating rate & 1.05 \\
Fixed rate & 0.42 \\
\hline Trust Type & \\
Mortgage Loan Trust & 0.83 \\
Other Trust & 0.83 \\
\hline Initial rating & \\
AAA & 0.43 \\
AA & 0.75 \\
A & 1.11 \\
BBB & 1.81 \\
BB and below & 1.92 \\
\hline Parent Rating & \\
AAA & 0.96 \\
AA & 0.78 \\
A & 0.82 \\
BBB & \\
BB and below & 0.85 \\
NR & 0.80 \\
\hline Deal Participants & 0.77 \\
Sponsor $=$ servicer & 0.68 \\
Sponsor $=$ servicer $=$ underwriter \\
Servicer or underwriter unidentified & 0.91 \\
Sponsor $\neq$ servicer & 0.73 \\
\hline Sponsor Diversification & 0.73 \\
Collateral types issued $>4$ & 0.85 \\
Collateral types issued $\leq 4$ & 0.92 \\
\hline Whole Sample & \\
& \\
\hline & \\
\hline
\end{tabular}


Preliminary and Incomplete - Please do not quote or cite.

\section{Appendix 3: Coupon Spread Coefficients Not Reported in Table 1}

\begin{tabular}{|c|c|}
\hline Variables & Coupon Spread (ppt.) \\
\hline Vintage - 2000H1 & $\begin{array}{c}-0.618 * * * \\
(0.161)\end{array}$ \\
\hline Vintage - $2000 \mathrm{H} 2$ & $\begin{array}{c}0.000 \\
(0.000)\end{array}$ \\
\hline Vintage - 2001H1 & $\begin{array}{l}-0.018 \\
(0.126)\end{array}$ \\
\hline Vintage - 2001H2 & $\begin{array}{c}0.047 \\
(0.137)\end{array}$ \\
\hline Vintage - 2002H1 & $\begin{array}{c}0.087 \\
(0.130)\end{array}$ \\
\hline Vintage - 2002H2 & $\begin{array}{l}-0.161 \\
(0.123)\end{array}$ \\
\hline Vintage - 2003H1 & $\begin{array}{c}-0.389 * * * \\
(0.127)\end{array}$ \\
\hline Vintage - $2003 \mathrm{H} 2$ & $\begin{array}{c}-0.280 * * \\
(0.126)\end{array}$ \\
\hline Vintage - 2004H1 & $\begin{array}{c}-0.325^{* * *} \\
(0.124)\end{array}$ \\
\hline Vintage - 2004H2 & $\begin{array}{c}-0.494 * * * \\
(0.119)\end{array}$ \\
\hline Vintage - 2005H1 & $\begin{array}{c}-0.872 * * * \\
(0.118)\end{array}$ \\
\hline Vintage - 2005H2 & $\begin{array}{c}-1.033 * * * \\
(0.117)\end{array}$ \\
\hline Vintage - 2006H1 & $\begin{array}{c}-1.102 * * * \\
(0.119)\end{array}$ \\
\hline Vintage - $2006 \mathrm{H} 2$ & $\begin{array}{c}-1.080 * * * \\
(0.122)\end{array}$ \\
\hline Vintage - 2007H1 & $\begin{array}{c}-0.954 * * * \\
(0.121)\end{array}$ \\
\hline Vintage - 2007H2 & $\begin{array}{c}-0.800 * * * \\
(0.135)\end{array}$ \\
\hline Vintage - 2008H1 & $\begin{array}{c}0.526^{* * *} \\
(0.200) \\
\end{array}$ \\
\hline
\end{tabular}

\begin{tabular}{lc} 
Variables & Coupon Spread (ppt.) \\
\hline Collateral type - Auto loans & $0.428^{* * *}$ \\
& $(0.127)$ \\
Collateral type - Equipment & 0.132 \\
& $(0.219)$ \\
Collateral type - Home equity & $0.690^{* * *}$ \\
& $(0.115)$ \\
Collateral type - RMBS & $0.720^{* * *}$ \\
& $(0.113)$ \\
Collateral type - Student loans & 0.285 \\
& $(0.180)$ \\
\hline Constant & $1.293 * * *$ \\
& $(0.169)$ \\
Observations & 88288 \\
R-squared & 0.170 \\
\hline
\end{tabular}

Robust standard errors in parentheses

Standard errors clustered by deal

$* * * \mathbf{p}<0.01, * * \mathbf{p}<0.05, * \mathbf{p}<0.1$ 
Preliminary and Incomplete - Please do not quote or cite.

\section{Appendix 4: Coefficients from Coupon Spread and Hazard Regressions}

\begin{tabular}{|c|c|c|}
\hline Seller & Hazard & Spread \\
\hline ACC CAPITAL HOLDINGS & -0.01 & -0.18 \\
\hline AMERICAN HOME MORTGAGE INVESTMENT & 0.02 & 0.17 \\
\hline AMERICREDIT & -0.27 & 0.24 \\
\hline BANK OF AMERICA & 0.13 & 0.30 \\
\hline BEAR STEARNS & 0.67 & -0.53 \\
\hline CAPITAL ONE FINANCIAL CORP & -0.18 & 0.23 \\
\hline C-BASS & 0.20 & -0.11 \\
\hline CHEVY CHASE BANK & 0.22 & -1.03 \\
\hline CITIGROUP & 0.09 & -0.06 \\
\hline COUNTRYWIDE FINANCIAL CORP & 0.06 & 0.08 \\
\hline CREDIT SUISSE GROUP & 0.02 & 0.46 \\
\hline DEUTSCHE BANK & 0.03 & -0.03 \\
\hline FIRST HORIZON NATIONAL CORP & 0.11 & 0.65 \\
\hline FORD MOTOR COMPANY & 3.13 & -0.29 \\
\hline GMAC & -0.04 & -0.13 \\
\hline HONDA MOTOR & 2.26 & 0.03 \\
\hline JPMORGAN CHASE \& CO & 0.08 & 0.23 \\
\hline LEHMAN BROTHERS HOLDINGS & -0.04 & 0.12 \\
\hline MERRILL LYNCH AND CO & -0.03 & -0.03 \\
\hline MORGAN STANLEY & -0.09 & 0.10 \\
\hline NELNET & 2.49 & -0.65 \\
\hline NISSAN MOTOR & 2.20 & 0.12 \\
\hline REDWOOD TRUST & 0.15 & -0.99 \\
\hline ROYAL BANK OF SCOTLAND GROUP & -0.04 & -0.15 \\
\hline SLM HOLDING CORP & 0.56 & -0.65 \\
\hline THORNBURG MORTGAGE & 0.27 & -0.33 \\
\hline UBS & 0.07 & 0.24 \\
\hline WASHINGTON MUTUAL & 0.05 & 0.23 \\
\hline WELLS FARGO & 0.18 & 0.37 \\
\hline WESTCORP & 2.18 & -0.37 \\
\hline
\end{tabular}

